Article published in Epilepsy Behav. 2019 Jan;90:209-216. doi: 10.1016/j.yebeh.2018.11.035

\title{
Therapeutic itinerary of children living with epilepsy in Kinshasa: features, determinants and relationships with behavioral problems and cognitive impairment
}

Thierry Matonda-ma-Nzuzi ${ }^{\mathrm{a}, *}$, Samuel Mampunza Ma Miezi ${ }^{\mathrm{a}}$, Dominique Charlier-Mikolajczak ${ }^{\mathrm{b}}$, Diane Muanza Mvumbi ${ }^{a}$,Fanny Malendakana ${ }^{c}$, Glennie Ntsambi Eba ${ }^{\mathrm{d}}$, Damien Junior Mayemba Nsundi $^{a}$, Davin Mpaka Mbeya ${ }^{\mathrm{a}}$, Magloire Nkosi Mpembi ${ }^{\mathrm{a}}$, Gilbert Mananga Lelo ${ }^{\mathrm{e}}$.

aDepartment of Psychiatry, Faculty of medicine, Université de Kinshasa, Democratic Republic of the Congo

bervice of child psychiatry, Faculty of medicine, Université catholique de Louvain, Belguim 'Service of Pediatrics, Far East Rand Hospital, Johannesburg, South Africa.

${ }^{\mathrm{d}}$ Unity of neurosurgery, Department of surgery, Faculty of medicine, Université de Kinshasa, Democratic Republic of the Congo

e Department of Neurology, Faculty of medicine, Université de Kinshasa, Democratic Republic of the Congo

Corresponding author: Thierry Matonda-ma-Nzuzi, MD, Departement of Psychiatry, Centre NeuroPsychopathologique de l'Université de Kinshasa, Faculty of Medecine, Université de Kinshasa, Lemba, Kinshasa XI, Democratic Republic of the Congo. matondathierry@yahoo.fr

\begin{abstract}
Background: Epilepsy mostly affects children in sub-Saharan Africa. However, little is known about the therapeutic itinerary of these children living with epilepsy (CWE). This study aims to describe the therapeutic itinerary of CWE in Kinshasa and to analyze its relationships with clinical features, behavioral problems and cognitive impairment.

Methods: This hospital-based study has included $104 \mathrm{CWE}$ aged 6 to 17 years. The features of their therapeutic itinerary and their relationship with clinical features, behavioral problems and cognitive impairment were analyzed.

Results: The vast majority of CWE (87\%) has started their therapeutic itinerary by the Western medicine. The first source of information about epilepsy as well as the type of antiepileptic treatment varied with the socioeconomic status of families of CWE. The total duration of the therapeutic itinerary was shorter for the CWE who were living with both their parents $(\mathrm{P}=.038)$, who had generalized
\end{abstract}


seizures $(\mathrm{P}=.0073)$ or who had no family history of epileptic seizures $(\mathrm{P}=.019)$. The $\mathrm{CWE}$ who had total behavioral problem, compared to the others, were putting more time $(\mathrm{P}=.021)$ to reach the CSMT after the suspicion or the diagnostic of epilepsy. The total duration of CWE who had cognitive impairment $(\mathrm{P}=.021)$ was longer than that of $\mathrm{CWE}$ who had not cognitive impairment.

Conclusion: the therapeutic itinerary of CWE in Kinshasa began with Western medicine. The remainder of this therapeutic itinerary highlights the difficulties of the primary level of the pyramid of care in the management of these CWE. This study also shows that the therapeutic itinerary of CWE was associated with socioeconomic conditions, clinical features, behavioral problems and cognitive impairment. Key words : Epilepsy; Children with epilepsy; Therapeutic itinerary; Cognitive impairment; Behavioral problems; sub-Saharan Africa.

\section{Introduction}

Analysis of the therapeutic itinerary is important to understand the determinants of the quest of the care for a disease. In sub-Saharan Africa, only a small number of studies have been devoted to the therapeutic itinerary of people living with epilepsy (PWE). These studies describe a therapeutic itinerary of PWE that passes successively or simultaneously through three types of treatment: Western medicine, traditional medicine and prayer [1-4].

Traditional medicine is often the first step in the quest for care of sub-Saharan Africa's PWE [2,3,5]. Its geographical, economic and cultural accessibility pleads in its favor [3,5]. In sub-Saharan Africa treatment with Western medicine is not the first choice of PWE $[2,3,5,6]$. Only one-third of PWE arriving in a neurological structure has ever received antiepileptic medication [6-8]. Although very little documented, prayer is also used as a therapeutic means $[1,2,4]$.

The therapeutic itinerary of PWE in sub-Saharan Africa is responsible for late arrival of patients in appropriate health facilities [6]. Birbeck [6] has found that the PWE in sub-Saharan Africa take up to 4.7 years to reach an appropriate structure for the care of epilepsy. This delay has consequences on clinical characteristics, cognitive performances and the psychic functioning of the PWE. For children, for example, delay in the diagnosis, and consequently in the management of the epilepsy, aggravates it [9].

While epilepsy, in sub-Saharan Africa, more frequently affects children [8,10,11], we have not found studies specifically devoted to the therapeutic itinerary of children living with epilepsy (CWE). Thus, this study aims to describe the therapeutic itinerary of CWE, to identify its determinants and to analyze its relationships with behavior problems and cognitive impairment. This study is complementary to another one on behavioral problems and cognitive impairment in CWE of Kinshasa [12]. 


\section{Methods}

\subsection{Nature, period of study and study setting}

The present study is a cross-sectional hospital-based study. The inclusion of CWE, by a convenience sampling, was done in two times, from April to May 2013 and from November to December 2013. The 'Centre de Santé Mental Telema' (CSMT or Mental Health Center Telema) located in the city of Kinshasa (capital of the Democratic Republic of the Congo) was the framework of this study. This neurological and psychiatric of ambulatory care health center is situated in the eastern part of the city of Kinshasa. Its geographic location, the relatively low cost of the care and the high influx of person with epilepsy are its main assets [12]. The CSMT performs more than 30,000 consultations annually. It has 2 EEG-videos and a pharmacy that sells psychotropic drugs at a low price.

\subsection{Children with epilepsy}

This study has included active CWE aged 6 to 17 years. These CWE had presented at least two unprovoked epileptic seizures separated by a 24 hours interval, the last of which had occurred in the last 5 years [13]. Seizures have been classified according to the recommendations of the International League Against Epilepsy [14]. The description of seizures was collected from eye witnesses of epileptic seizures.

\subsection{Characteristics of the therapeutic itinerary}

The determinants of the therapeutic itinerary have been analyzed from four variables: the types of consultations prior to the first arrival at the CSMT, the first source of suspicion or of diagnosis of epilepsy, the type of antiepileptic treatment prior to the first consultation at the CSMT and the duration of the therapeutic itinerary.

The types of consultations prior to the first arrival at CSMT included Western medicine (regardless of care provider quality), traditional medicine and prayer. The CWE for which information were lacking to fit into one of these three categories were included in category named "other".

The first source of suspicion or of diagnosis of epilepsy targeted the first source who had suspected or diagnosed epilepsy as the children's disease. It could be family or relatives, a care provider of Western medicine or a traditional healer. In this study, the family is understood in the broad sense and not in the sense of the nuclear family.

The type of antiepileptic treatment prior to the first consultation at the CSMT was considered. The types of antiepileptic treatment were including: antiepileptic drugs (AEDs), traditional therapy (TT), and the combination of AEDs and TT (AEDs-TT). In this study, the combination does not require the simultaneous use of both types of treatment. 
The duration of the therapeutic itinerary was divided into three: i) the duration I : between the first seizure and the suspicion or the diagnosis of epilepsy; ii) the duration II : between the suspicion or the diagnosis of epilepsy and the first consultation at the CSMT; iii) and the total duration between the first epileptic seizure and the first consultation at the CSMT.

\subsection{Sociodemographic variables}

The following variables were taken into account: the age, the gender, the family type (child living with both parents or other), the father's age, the mother's age, the household size, the estimation of the equivalent amount of money in United States dollars (\$) usually spent on daily household needs and per household member.

\subsection{Epileptic features}

The following variables were considered: the age at first seizure, the history of epileptic seizure in the family, the type of epileptic seizures and the estimated monthly epileptic seizure frequency. The type of epileptic seizures was dichotomized into generalized and other seizures (focal seizures and spasms). The unclassified crises were omitted from statistical analyzes. The monthly seizure frequency was categorized in two: $<2$ seizures per month and $\geq 2$ seizures per month.

\subsection{Behavioral problems}

The behavioral assessment was performed with the Asselbach child behavior checklist (CBCL). The behavioral problems (internalized, externalized and total) in the CWE included in this study were the subject of a previous publication [12].

\subsection{Cognitive impairment}

The cognitive performance was assessed using the short version (2 subtests) of the Wechsler Nonverbal (WNV) scale of ability. The cognitive impairment was defined as a score of less than 70 on the WNV or the inability of the CWE to respond to WNV items coupled with a clinical history of intellectual disability. The cognitive impairment of CWE included in this study was the subject of a previous publication [12].

\subsection{Missing data}

To complete missing informations for some CWE, according to the case, we conducted telephonic calls or we used medical files for CWE followed at the CSMT [12]. Missing data are indicated in the tables of results.

\subsection{Statistical analyses}

The analyses of the data were performed with the softwares Statistica 13 and SPSS 20. Associations between categorical variables were searched by the Pearson chi-square test eventually followed by 
analysis of standardized residuals as « post hoc » after Bonferroni corrections $\left(\alpha_{\text {cor }}=0,05 /\right.$ (number of rows $\mathrm{X}$ number of columns)).

The normality of the quantitative data was assessed by the Shapiro test. The homoscedasticity was evaluated by the Levene's test. Parametric statistical tests were used for quantitative variables that have met at least equality of variances and did not exhibit important asymmetry. For the previous antiepileptic treatment and the source of the suspicion or the diagnostic of epilepsy, the means of groups were compared by planned orthogonal linear contrast tests (see supplemantary materials). Nonparametric tests were performed for quantitative variables that did not meet at least the equality of variances or had exhibited a high asymmetry. Mann-Whitney U-tests and Kruskal Wallis test, eventually followed by multiple comparisons of mean ranks, and Spearman correlations were used depending on the situation.

The graphics have been plotted with software R 3.1.2. (R Core Team, 2014).

\subsection{Ethical approval}

The present research has obtained the agreement of the Ethics Committee of the School of Public Health of the University of Kinshasa (ESP/CE/018/13). The inclusion in the study was conditioned upon the signing of an informed consent form by an adult accompanying the CWE.

\section{Results}

\subsection{Characteristics of CWE}

This study had included 104 CWE (table 1). Their characteristics were described previously [11].

Table 1. General characteristics of CWE

\begin{tabular}{lc}
\hline Variable & $\mathrm{n}(\%)$ or mean $\mathbf{S D}$ \\
\hline Age, years & $12.0 \pm 3.2$ \\
Male & $61(58.6 \%)$ \\
Father's age, years $(\mathrm{N}=91)$ & $48.9 \pm 8.0$ \\
Mother's age, years $(\mathrm{N}=97)$ & $41.5 \pm 7.0$ \\
Lives with both parents $(\mathrm{N}=98)$ & $74(75.5 \%)$ \\
Size of household $(\mathrm{N}=102)$ & $7.2 \pm 2.6$ \\
Household daily expenditures, \$ (N=98) & $8.04 \pm 4.14$ \\
Individual daily expenditures, \$ $(\mathrm{N}=98)$ & $1.24 \pm 0.72$ \\
History of epileptic seizure in family & $42(40.4 \%)$ \\
Age of onset, (years) & $6.9 \pm 4.0$ \\
Generalized seizure $(\mathrm{N}=96)$ & $35(36.4 \%)$ \\
$\geq 2$ seizure/month $(\mathrm{N}=103)$ & $59(57.3 \%)$ \\
Cognitive impairment $(\mathrm{N}=90)$ & $66(73.3 \%)$ \\
Internalized behavioral disorder & $24(23.1 \%)$ \\
Externalized behavioral disorder & $28(26.9 \%)$ \\
Total behavioral disorder & $30(28.8 \%)$ \\
\hline
\end{tabular}




\subsection{Characteristics of the therapeutic itinerary}

The diagram 1 traces and details the therapeutic itinerary of 101 CWE. The Western medicine was the first step of treatment for $88(87.1 \%)$ CWE. These CWE who had started with Western medicine then moved on to the CSMT (35/88 or 39.8\%), to a traditional healer (33/88 or $37.5 \%$ ) or to a prayer (19/88 or $21.6 \%$ ). Thus, $59.1 \%$ (52/88) families of the CWE seen in the health facilities have sought thereafter for the healing based on beliefs. Both the traditional medicine and the prayer were the first consultation for five CWE (5.0\%). The CSMT was the first step of the therapeutic itinerary for only one child living with epilepsy (1.0\%). One of the two children who had started their therapeutic itinerary with an "other" structure had taken an antiepileptic drug by self-medication.

Finally, during their therapeutic itinerary, 92 (91.1\%), 45 (44.6\%) and 34 (33.7\%) CWE have passed, respectively, towards the Western medicine, the traditional medicine and the prayer, before arriving at the CSMT. Overall, Western medicine had preceded, directly or indirectly, belief-based treatment in 67 (66.3\%) CWE, with traditional medicine for 39 (38.6\%) CWE, and prayer for 28 (27.7\%) CWE. During their therapeutic itinerary, the CWE have consulted successively up to four different types of consultations (Table 2). Three quarters (76.3\%) of the CWE had arrived at the CSMT with no suspicion or diagnosis of epilepsy (43.3\%) or with suspicion from family or relatives $(33.0 \%)$. The majority (55.9\%) of the CWE had previously received antiepileptic treatment with antiepileptic drugs (13.7\%), traditional medicine $(29.4 \%)$ or both (12.8\%). Thus $26.5 \%$ of CWE had previously received antiepileptic drug and $42.2 \%$ a traditional treatment. 
Table 2. Characteristics of the therapeutic itinerary

\begin{tabular}{lc}
\hline Variable & $\mathrm{n}(\%)$ or median (IQR) \\
\hline Number of type of previous consultation (101) & $1(1.0 \%)$ \\
- None & $41(40.6 \%)$ \\
- One & $39(38.6 \%)$ \\
- Two & $19(18.8 \%)$ \\
- Three & $1(1.0 \%)$ \\
- Four & \\
First source of suspicion/diagnostic (N=97) & $42(43.3 \%)$ \\
- None & $32(33.0 \%)$ \\
- Family/relatives & $8(8.2 \%)$ \\
- Traditional healer & $15(15.5 \%)$ \\
- Health facilities & \\
Type of previous antiepileptic treatment (N=102) & $45(44.1 \%)$ \\
- None & $30(29.4 \%)$ \\
- Traditional treatment & $13(12.7 \%)$ \\
- Antiepileptic drugs+Traditional treatment & $14(13.7 \%)$ \\
- Antiepileptic drugs & \\
Duration & $1(0-2.5)$ \\
- Duration I (year) & $0(0-2.5)$ \\
- Duration II (year) & $2(1-6)$ \\
- Total duration (years) &
\end{tabular}

CSMT: Centre de Santé Mentale Telema.

The suspicion or the diagnosis of epilepsy had occurred $1(0-2.5)$ year after the first seizure. Thereafter, the CWE took less than a year (0 [0-2.5] year) to arrive at the CSMT. The total duration of the therapeutic itinerary, from the first epileptic seizure to the arrival to the CSMT, was 2(1-6) years.

\subsection{Reciprocal relationship between the therapeutic itinerary features}

There was a statistically significant relationship between the first source of suspicion or diagnosis of epilepsy and the type of treatment (Khi2 $=40.4, \mathrm{df}=9, \mathrm{P}=.00001$; table 3 ). The threshold $\alpha$ for the post hoc assessment to independence deviations was of $0.05 /(4 \mathrm{X} 4)$ or 0.003125 . The CWE who had been diagnosed by traditional healers were more likely to receive traditional treatment $(\mathrm{P}=.0000048)$. And the CWE who had been diagnosed in health facilities were more likely to receive AED alone $(\mathrm{P}=$. 0026). 
Table 3. First source of suspicion/diagnostic and antiepileptic treatment (with their adjusted residuals)

\begin{tabular}{llllll}
\hline & \multicolumn{4}{c}{ First source of suspicion or diagnostic } & \\
\cline { 2 - 5 } & None & Family/Relatives & TH & HF & P \\
\hline Antiepileptic treatment & & & & & $<.001$ \\
None & $25(2.9)$ & $13(-0.6)$ & $0(-2.7)$ & $5(-1.0)$ & \\
Traditional & $8(-1.7)$ & $11(0.7)$ & $\mathbf{8}(\mathbf{4 . 6})$ & $1(-2.1)$ & \\
AED+Traditional treatment & $1(-2.2)$ & $6(1.9)$ & $0(-1.0)$ & $3(1.3)$ \\
AED & $6(0.1)$ & $2(-1.7)$ & $0(-1.2)$ & $\mathbf{6 ( 3 . 0 )}$ & \\
\hline
\end{tabular}

AED: antiepileptic drug. TH: traditional healer. HF: Health faliclity.

The duration I did not vary with the source of the suspicion or the diagnosis of epilepsy $(\mathrm{H}(3, \mathrm{~N}=97)$ $=0.9, \mathrm{P}=.82$ ). There was a relationship between the source of the suspicion or the diagnosis of epilepsy and the duration II of the therapeutic itinerary $(\mathrm{H}(3, \mathrm{~N}=97)=48.7, \mathrm{P}<.001$, figure $1 \mathrm{~A})$. The group of CWE who had arrived at the CSMT without suspicion or diagnosis of epilepsy (0 (0-0) year) have shorter duration II than those who had the suspicion or the diagnosis by relatives or family (2 (14) years, $\mathrm{P}<.000001)$, by traditional healer $(2(0.5-4.5)$ years, $\mathrm{P}=.0073)$ or Western medicine $(1(0-5)$ year, $\mathrm{P}=.0048)$. The total duration of the therapeutic itinerary was varied according to the source of suspicion or diagnosis of epilepsy $(\mathrm{H}(3, \mathrm{~N}=97)=15.2, \mathrm{P}=.001$, figure $1 \mathrm{~B})$. The CWE who did not had suspicion or diagnosis of epilepsy (1 (0-2) year) were arriving faster at the CSMT than the CWE for whom suspicion was emanating from family or relatives $(4(2-8)$ years, $\mathrm{P}=.0021)$.

The duration I did not vary with the type of the previous treatment $(\mathrm{H}(3, \mathrm{~N}=102)=3.7, \mathrm{P}=.29)$.

There was a relationship between the type of the previous treatment and the duration II of the therapeutic itinerary $(\mathrm{H}(3, \mathrm{~N}=102)=14.7, \mathrm{P}=.002$, figure $1 \mathrm{C})$. The $\mathrm{CWE}$ who had not previously received antiepileptic treatment (0 (0-0) year) had a duration II shorter than the CWE treated with AED combined to traditional treatment $(2(1-3)$ years; $\mathrm{P}=.041)$ and than the CWE treated with traditional treatment alone $(1(0-4)$ year, $\mathrm{P}=.027)$. The total duration was also varied with the types of antiepileptic treatment $(\mathrm{H}(3, \mathrm{~N}=102)=8.5, \mathrm{P}=.037$, figure $1 \mathrm{D})$. But the multiple comparisons of mean ranks had fallen to found which groups were different.

\subsection{Analysis of characteristic of the therapeutic itinerary according to sociodemographic characteristics and characteristics of epilepsy}

The per capita daily expenditures of the CWE's families without suspicion nor diagnosis of epilepsy $(1.45 \pm 0.70 \$)$ were higher $(\mathrm{t}=2.1, \mathrm{P}=.035$, figure $2 \mathrm{~A})$ than those of families of the CWE who had been suspected or diagnosed by the traditional healers $(1.00 \pm 0.66 \$)$ combined with to family or the relatives $(1.09 \pm 0.73 \$)$. 
The families of CWE previously traditionally treated had daily expenditures per household $(6.42 \pm 3.26$ $\$$ versus $8.80 \pm 3.97 \$, \mathrm{t}=2.4, \mathrm{P}=.018)$ and per individual $(0.99 \pm 0.58 \$$ versus $1.37 \pm 0.71 \$, \mathrm{t}=2.2$, $\mathrm{P}=.029$ ) lower than those of previously untreated CWE (Figures $2 \mathrm{~B}$ and $2 \mathrm{C}$ ).

The CWE who were living with their both parents had a shorter duration I than the other CWE (1 (0-2) year versus $2(1-5)$ years, $\mathrm{N}=98, \mathrm{U}=550.5, \mathrm{P}=.0037$, figure $2 \mathrm{D})$. The duration I was shorter for the CWE with generalized seizures than for the CWE with other types of seizures $(0(0-1)$ year versus $1(0$ 4) year, $\mathrm{N}=96, \mathrm{U}=697.0, \mathrm{P}=.0032$, figure $2 \mathrm{E}$ ). Duration I was negatively correlated with age at first epileptic seizure $(\rho=-0.41, P=.000016$, table 4$)$.

Table 4. Matrix of correlations of the durations of the therapeutic itinerary

\begin{tabular}{lllllllll}
\hline & Age & \multicolumn{3}{c}{ Age of onset } & \multicolumn{2}{c}{ DEH } & \multicolumn{3}{c}{ DEI } \\
& $\rho$ & $\mathrm{P}$ & $\rho$ & $\mathrm{P}$ & $\rho$ & $\mathrm{P}$ & $\rho$ & $\mathrm{P}$ \\
\hline Duration I & 0.179 & .068 & -0.409 & $<.001$ & 0.066 & 0.52 & 0.171 & .093 \\
Duration II & 0.218 & .026 & -0.210 & .032 & -0.225 & .026 & -0.260 & .0097 \\
Total duration & 0.329 & $<.001$ & -0.539 & $<.001$ & -0.163 & .11 & -0.097 & .34 \\
\hline
\end{tabular}

DEH: Daily expenditures per household. DEI: Daily expenditures per individual

The duration II was positively correlated with age $(\rho=0.22, P=.026$, table 4$)$. This duration was negatively correlated with the age at the first seizure $(\rho=-0.21, \mathrm{P}=.032)$, the family's daily expenditures per household $(\mathrm{N}=98, \rho=-0.22, \mathrm{P}=.026)$ and per individual of household $(\mathrm{N}=98, \rho=$ $-0.26, \mathrm{P}=.0097)$.

The total duration was shorter for CWE who were living with their both parents (2 (1-5) years versus 4 (1.5-9) years, $\mathrm{N}=98, \mathrm{U}=638.5, \mathrm{P}=.038$, figure $2 \mathrm{~F})$, who had generalized seizures (1 (0-4) year versus 3 (1-7) years, $\mathrm{N}=96, \mathrm{U}=718.5, \mathrm{P}=.0073$, figure $2 \mathrm{G}$ ) or who did not have a history of epilepsy in their family (1 (1-5) year versus 3 (2-8) years, $\mathrm{U}=950.0, \mathrm{P}=.019$, figure $2 \mathrm{H}$ ). The total duration was positively correlated with age $(\rho=0.33, P=.00064$, table 4$)$ and negatively correlated with age at first epileptic seizure $(\rho=-0.54, P<.000001)$.

\subsection{Relationship between the therapeutic itinerary, the behavioral problems and the cognitive impairment}

There was a relationship between internalized behavioral disorder and the first source of suspicion or diagnosis of epilepsy (Chi-square $=8.5, \mathrm{df}=3, \mathrm{P}=.036$; see supplemantary materials). Compared to other groups, the proportion of the internalized disorder was higher among the CWE who had been suspected of having epilepsy by family or by relatives $(P=.0060 ; \alpha$ corrected $0.05 /(4 X 2)=0.00625)$. The CWE with a total behavioral disorder had a duration II (1.5 (0-4) year versus $0(0-2.0)$ year, $\mathrm{U}=$ $821.5, \mathrm{P}=.021)$ greater than those of the $\mathrm{CWE}$ without total behavioral disorder (Figures $3 \mathrm{~A}$ ). 
The CWE with cognitive impairment had a total duration longer than those of CWE who did not have cognitive impairment (2 (1-6) years versus $1(0-4)$ years, $\mathrm{N}=90, \mathrm{U}=541.0, \mathrm{P}=.021$, and figure 3B).

\section{Discussion}

\subsection{Characteristics of the therapeutic itinerary}

The first goal of this study was to describe the therapeutic itinerary of CWE. The results of this study, as observed by very few authors [15], have shown that the first consultation unfold mainly in a Western medicine structure. This result is in contradiction with the vast majority of data from the sub-Saharan Africa literature describing therapeutic itinerary that begins with traditional medicine or prayer [1-3]. These studies have included only adults [1,2] or both adults and children [3]. The therapeutic itinerary of CWE could differ from that of adults living with epilepsy. Probably at the first consultation the CWE are treated as suffering from others common diseases in Africa, such as cerebral malaria and meningoencephalitis, which can be manifested by epileptic seizures. It has been observed that some CWE were treated with antimalarials and/or antibiotics $[7,16]$.

After the first type of consultation, the therapeutic itinerary was closer to that described in the subSaharan Africa literature [2]. The trigger for this change would be the suspicion or the diagnosis of epilepsy. Indeed, in sub-Saharan Africa, Epilepsy is considered as having a spiritual origin $[1,15]$. In Kinshasa, epilepsy is described by the population as "maladi ya ndeke" (bird disease). This name does not apply to epileptic seizures of others clinical entities such as cerebral malaria or meningitis. Aujoulat et al. [17] have shown that in sub-Saharan Africa the name of a disease influences the choice of the type of treatment. These researchers have observed that according to its name the same disease is treated either by Western medicine or by traditional treatment. This could explain why the families of children with epilepsy who had the information by the family or by the relatives had turned more often to traditional medicine. Conversely, families of children with epilepsy who did not suspect "maladi ya ndeke" as the disease of their child went to the CSMT or to other health facilities.

As elsewhere in sub-Saharan Africa, the total duration of the therapeutic itinerary of CWE observed in this study was very long. It was similar to the average of 2.2 years duration observed by DoumbiaOuattara et al. [3] in Ivory Coast. Moreover, these authors have described that PWE spend up to 3.6 years before taking an antiepileptic drug. But the duration of the therapeutic itinerary observed in our study was much lower than the 4.7 years observed in Zambia [6].

This therapeutic itinerary beginning in Western medical structures and then moving on to antiepileptic treatments based on beliefs, highlights the inability of health facilities to diagnose and to manage epilepsy. This motivates the struggle of many researchers in sub-Saharan Africa for a better training of health care workers at the primary level of the health care pyramid [18]. 


\subsection{Relationships between the characteristics of the therapeutic itinerary}

The results of this study have shown that the characteristics of the therapeutic itinerary were closely associated between them. They express the different facets of the therapeutic itinerary. These associations could be summed up, in a simplistic way, the more the first source of information about the disease was not accurate, the more families consulted many actors and took different types of treatment and this was lengthening the time of the course before reaching a care structure, appropriate to manage epilepsy [1]. For example, Koba Bora et al. [7] in Lumbumbashi, another city of the DRC, have noted that PWE spent fifteen months between their first visit to a traditional healer and their arrival in an appropriate health facility. And Birbeck [6], in Zambia, has found that the 4.7 years average duration of therapeutic itinerary grows to 9 years for the PWE who have resorted care to traditional healers.

\subsection{Therapeutic itinerary, sociodemographic and clinical characteristics}

This study has observed, like many other studies, that the type of antiepileptic treatment was associated with the socioeconomic conditions of the CWE's families [3,7,10,15]. The socioeconomic conditions were one of the main factors that were guiding the choice of the traditional treatment. Families of CWE who had benefited from the traditional treatment had less financial means and could not equally afford the current antiepileptic treatment. Matonda et al. [16] have found that these CWE had clinical outcomes more severe than the CWE that did not received antiepileptic treatment prior their arrival at the CSMT.

Few factors were associated with the duration I. As observed by others authors, this duration was reducing with generalized seizures (9) and was increasing with an early onset [1]. The children who were living with both their parents had suspicion or diagnosis of epilepsy more rapidly. The reason is not clear to us. Perhaps the level of education would have played a role [1,3,9]. Is it because, in DRC, men have higher level of education than women? The CWE who were living with their fathers could thus receive the suspicion or diagnosis of epilepsy earlier. It may also be that keeping in touch with the families of both parents could increase the number of "experts" of the disease. Or the fact that the parents are together reduces the time for consultation and/or fundraising for CWE's care. Be that as it may, this has reduced the total length of the therapeutic itinerary for the children who were living with both parents.

In this study, several factors were associated with the duration II and the total duration of the therapeutic itinerary. Like other authors [1,9], this study has found that non-generalized seizures, young age, and early onset have lengthened the therapeutic itinerary. These variables reflect the difficulty of diagnosing epilepsy, particularly in toddlers [9]. Moreover, at this age, many conditions, such as 
cerebral malaria and meningitis, that can also manifest themselves by epileptic seizures may have increased the difficulty of diagnosing epilepsy.

The existence of a family history of epilepsy had lengthened, paradoxically, the total duration of the therapeutic itinerary. Probably those who had a history of epilepsy in the family were wasting time seeking treatment based on belief. Two elements could explain this paradox. On the one hand, it is difficult for sub-Saharan Africans to accept the chronicity of a disease as being natural [15,17]. And on the other hand, a failure, real or perceived as such, of a drug treatment in the other family member suffering from epilepsy may explain the gravitation towards belief-based treatment [15,17]. Moreover, the families do not exclude that epilepsy may be cured without the use of any antiepileptic drug, particularly for cultural believes $[10,15]$.

\subsection{Therapeutic itinerary, behavioral problems and cognitive impairment}

The results of this study showed that internalized behavioral disorders were more common among the CWE who had received the first information about their illness from a relative. Thus, the CWE would be sensitive to whoever first suspected or diagnosed their illness or to what was said to them the first time about epilepsy. Conversely, receiving the suspicion or the diagnosis by the different caregivers, from Western medicine as well as from traditional healers, does not increase the internalized behavior disorder more than the ignorance of the disease. These caregivers seem to be holding a communication or non-traumatic attitudes for the CWE. Particularly for traditional medicine, this finding echoes the observation of the authors who have examined the beneficial role of traditional healers on the psyche of patients [19]. Thus, one can conclude that the traditional medicine would have no side effect on the

psyche of CWE. But by lengthening of the duration of the therapeutic itinerary, the traditional medicine would have side effects on the other clinical features of CWE [6,9].

In this study, the CWE with total behavior problem compared to the other CWE had great duration II that had impacted the total duration of the therapeutic itinerary. These relationships seem to highlight the crucial role of the announcement of and the period after the suspicion or the diagnosis of epilepsy. This goes in the same sense that the association between the internalized behavioral problem and the first source of suspicion or diagnosis of epilepsy. Some factors associated to duration II, example socioeconomic situation, have been shown to favor behavior disorders [20].

Among different characteristics of the therapeutic itinerary, only the total duration of the course was associated with cognitive disorder. This result is consistent with that of researchers working on the consequences of delayed diagnostic and management of epilepsy [9]. Nevertheless, as discussed above, the duration of the therapeutic itinerary was associated with the other characteristics of the therapeutic itinerary. A small number of health actors, the lack of suspicion or diagnosis of epilepsy, and the 
absence of a prior antiepileptic treatment was reducing the length of therapeutic itinerary. Particularly for treatments with antiepileptic drugs and traditional, these findings are consistent with the findings of other researchers. People living with epilepsy lose a valuable time in medical structures and at traditional healers [6].

The relationship between the duration of the therapeutic itinerary with both the cognitive impairment and the behavioral problems is very interesting. This time was increased by, among others factors, the inability of the care providers of the first line to diagnose and to offer an appropriate treatment of epilepsy. Thus, the improvement of the ability of these care providers could reduce the occurrence of behavioral problems and of cognitive impairments.

\subsection{Limits}

This study is a hospital-based study and thus the CWE included in this study are not representative of all the CWE of Kinshasa. Nevertheless, most of our results are consistent with the literature data. Moreover, to our knowledge, this study is the first one devoted to the therapeutic itinerary of CWE. It raises several interrogations that could be subjects of others studies on the therapeutic itinerary of these children.

\section{Conclusion}

This study, contrariwise to the results of the vast majority of studies, has found that the therapeutic itinerary of the CWE has begun with Western medicine. This highlights the difficulties of the health structures to diagnose and to manage epilepsy. The rest of the therapeutic itinerary, with belief-based treatments, was similar to what is reported in the sub-Saharan literature. This therapeutic itinerary was closely related to the clinical characteristics and socioeconomic conditions in which live the CWE. This therapeutic itinerary was also associated with behavioral problems and cognitive impairment.

\section{Funding sources}

Dr Thierry Matonda-ma-Nzuzi is very grateful to the Fonds de Soutien Marie Marguérite Delacroix for their financial support.

\section{Disclosures}

The authors do not have any conflicts of interest to disclose.

\section{Acknowledgements}

We gratefully acknowledge the headmaster of CSMT for their collaboration. A big thanks to Professeur Jean Robert Rissassy Makulo and to Miss Cyrine Hmaied for their critical feedback.

\section{References}

[1] Bifftu BB, Dachew BA, Tiruneh BT, Alemu WG. First choice of treatment place in the pathways to epileptic care at the outpatient department of University of Gondar Hospital, Northwest Ethiopia: 
Cross-sectional institutional based study. PLoS One 2017;12(8):e0181310. doi:

10.1371/journal.pone.0181310.

[2] Ogunrin OA, Adeyekun A, Adudu P. Etiologies of epilepsy and health-seeking itinerary of patients with epilepsy in a resource poor setting: analysis of 342 Nigerian Africans. Seizure 2013;22(7):572-6. doi: 10.1016/j.seizure.2013.04.012.

[3] Doumbia-Ouattara M, Kouame-Assouan AE, Kouassi L, Diakite I, Boa YF, Sonan-Douayoua T. Itinéraire du patient épileptique reçu en consultation d'épileptologie à Abidjan. Rev Int Sc Méd 2013;15(2):69-73.

[4] Danesi MA, Adetunji JB. Use of alternative medicine by patients with epilepsy: a survey of 265 epileptic patients in a developing country. Epilepsia 1994;35(2):344-51.

[5] Osungbade KO, Siyanbade SL. Myths, misconceptions, and misunderstandings about epilepsy in a Nigerian rural community: implications for community health interventions. Epilepsy Behav 2011;21(4):425-9. doi: 10.1016/j.yebeh.2011.05.014.

[6] Birbeck GL. Seizures in rural Zambia. Epilepsia 2000;41(3):277-81.

[7] Koba Bora B, Lez DM, Luwa DO, Baguma MB, Katumbay DT, Kalula TK et al. Living with epilepsy in Lubumbashi (Democratic Republic of Congo): epidemiology, risk factors and treatment gap. Pan Afr Med J 2015;21:303. doi: 10.11604/pamj.2015.21.303.5580.

[8] Kariuki SM, Matuja W, Akpalu A, Kakooza-Mwesige A, Chabi M, Wagner RG et al. Clinical features, proximate causes, and consequences of active convulsive epilepsy in Africa. Epilepsia 2014;55(1):76-85. doi: 10.1111/epi.12392.

[9] Berg AT, Loddenkemper T, Baca CB. Diagnostic delays in children with early onset epilepsy: impact, reasons, and opportunities to improve care. Epilepsia 2014;55(1):123-32. doi:

10.1111/epi.12479.

[10] Sebera F, Munyandamutsa N, Teuwen DE, Ndiaye IP, Diop AG, Tofighy A et al. Addressing the treatment gap and societal impact of epilepsy in Rwanda--Results of a survey conducted in 2005 and subsequent actions. Epilepsy Behav 2015;46:126-32. doi: 10.1016/j.yebeh.2015.03.028.

[11] Munyoki G, Edwards T, White S, Kwasa T, Chengo E, Kokwaro G, Odera VM, Sander JW, Neville BG, Newton CR. Clinical and neurophysiologic features of active convulsive epilepsy in rural Kenya: a population-based study. Epilepsia 2010;51(12):2370-6. doi: 10.1111/j.15281167.2010.02653.x.

[12] Matonda-Ma-Nzuzi T, Mampunza Ma Miezi S, Mpembi MN, Mvumbi DM, Aloni MN, Malendakana F, Mpaka Mbeya D, Lelo GM, Charlier-Mikolajczak D. Factors associated with 
behavioral problems and cognitive impairment in children with epilepsy of Kinshasa, Democratic Republic of the Congo. Epilepsy Behav 2018;78:78-83. doi: 10.1016/j.yebeh.2017.08.030. [13] Commission on Epidemiology and Prognosis, International League Against Epilepsy. Guidelines for epidemiologic studies on epilepsy. Epilepsia 1993;34(4):592-6

[14] Berg AT, Berkovic SF, Brodie MJ, Buchhalter J, Cross JH, van Emde Boas W, Engel J, French J, Glauser TA, Mathern GW, Moshé SL, Nordli D, Plouin P, Scheffer IE. Revised terminology and concepts for organization of seizures and epilepsies: report of the ILAE Commission on Classification and Terminology, 2005-2009. Epilepsia 2010;51(4):676-85. doi: 10.1111/j.1528-1167.2010.02522.x. [15] Mushi D, Burton K, Mtuya C, Gona JK, Walker R, Newton CR. Perceptions, social life, treatment and education gap of Tanzanian children with epilepsy: a community-based study. Epilepsy Behav 2012;23(3):224-9. doi: 10.1016/j.yebeh.2011.12.003.

[16] Matonda-ma-Nzuzi T, Lelo GM, Mpembi Nkosi M, Madinga J, Kabwe Kola C, Maketa V, et al. Are the children with epilepsy treated traditionally a disadvantaged group? A pilot study. Pan Afr Med J 2016;23:229. doi:10.11604/pamj.2016.23.229.9165.

[17] Aujoulat I, Johnson C, Zinsou C, Guédénon A, Portaels F. Psychosocial aspects of health seeking behaviours of patients with Buruli ulcer in southern Benin. Trop Med Int Health 2003;8(8):750-9. [18] Kaputu-Kalala-Malu C. Epilepsies et crises épileptiques aiguës chez l'enfant en Afrique subsaharienne: défis et espoirs. Pan Afr Med J 2016;23:58. doi: 10.11604/pamj.2016.23.58.3273. [19] Abbo C, Ekblad S, Waako P, Okello E, Muhwezi W, Musisi S. Psychological distress and associated factors among the attendees of traditional healing practices in Jinja and Iganga districts, Eastern Uganda: a cross-sectional study. Int J Ment Health Syst 2008;2(1):16. doi: 10.1186/1752-44582-16.

[20] Carson J, Weir A, Chin RF, McLellan A. Socioeconomic deprivation is an independent risk factor for behavioral problems in children with epilepsy. Epilepsy Behav 2015;45:105-9.doi: 10.1016/j.yebeh.2015.03.009. 


\section{Diagram}

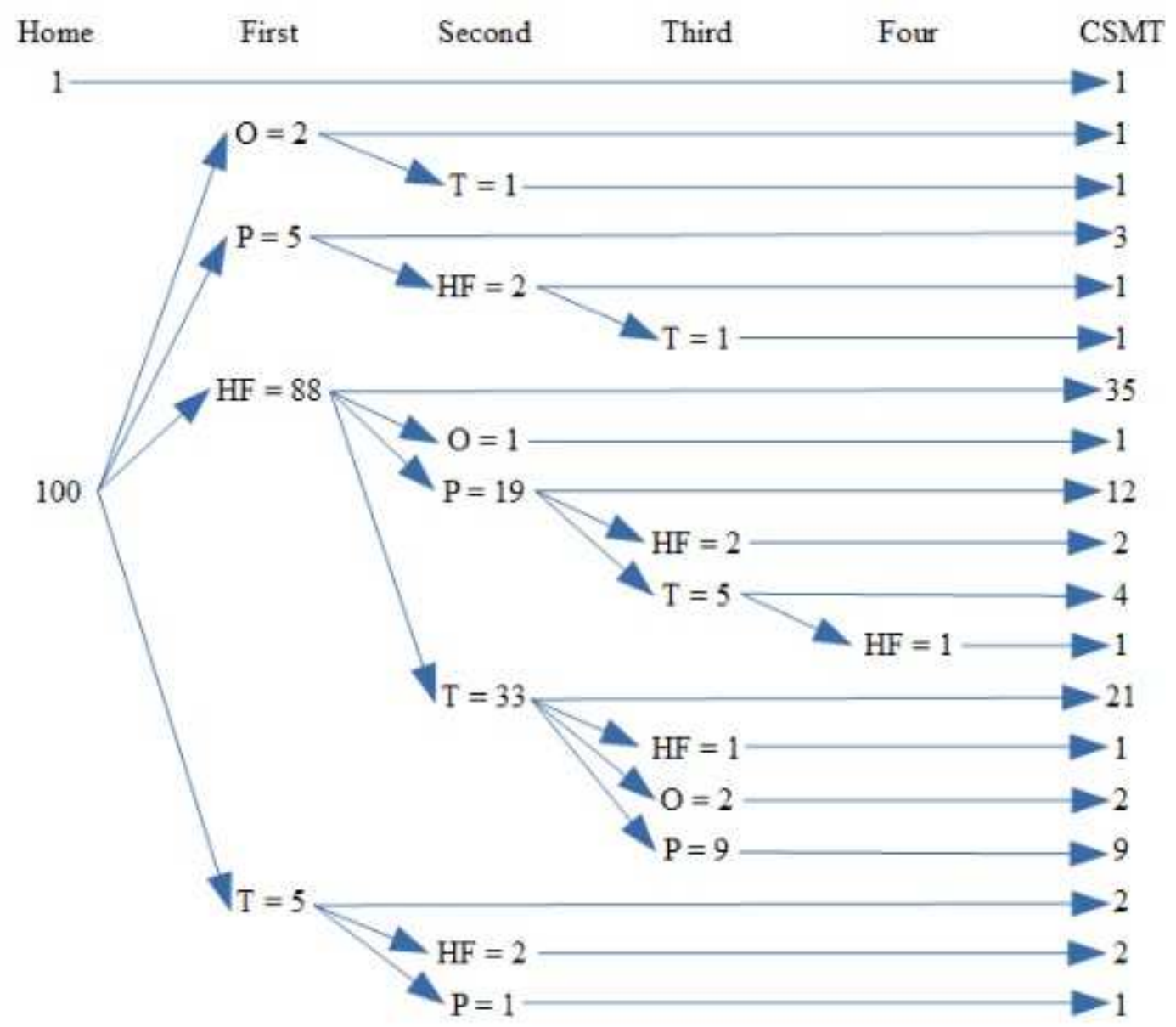

Diagram 1. Types of successive consultations. CSMT: Centre de santé mentale Telema.

HF: Health Facility. P: Prayer. O: Other. T: Traditional. 


\section{Figures}

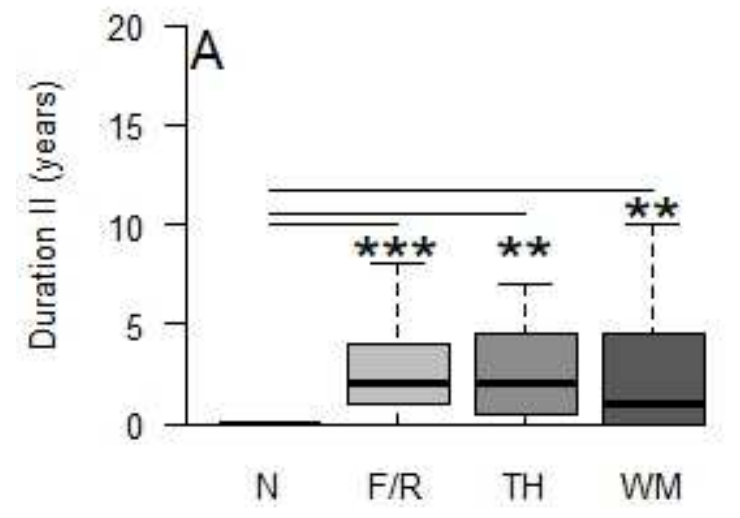

Source of suspicion/diagnostic

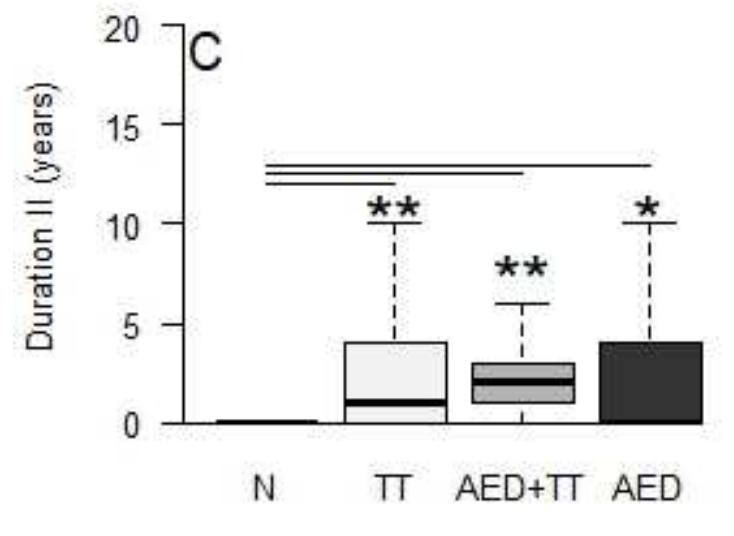

Previous antiepileptic treatment

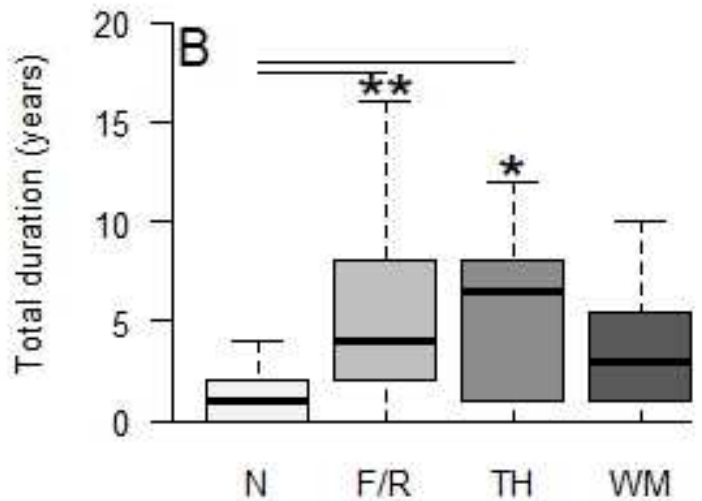

Source of suspicion/diagnostic

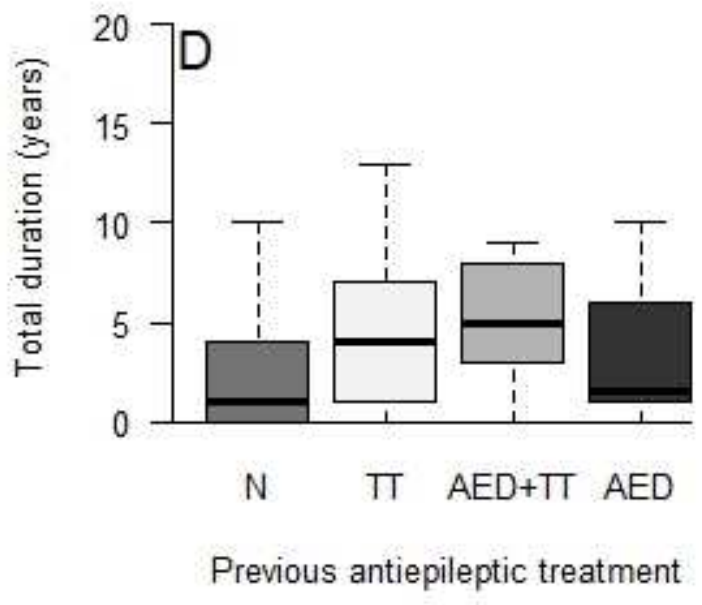

Fig. 1. Duration of the therapeutic itinerary according to the first source of suspicion or diagnosis of epilepsy and the type of antiepileptic treatment. $\mathrm{N}=$ none; $\mathrm{F} / \mathrm{R}=$ family or relatives; $\mathrm{TH}=$ Traditional healer; $\mathrm{WM}=$ Western Medicine; $\mathrm{TT}=$ traditional treatment $\mathrm{AED}+\mathrm{TT}=$ antiepileptic drug and traditional treatment; $\mathrm{AED}=$ antiepileptic drug. *: $\mathrm{P}$ b .05; **: $\mathrm{P} \mathrm{b} .01 ;$ ***: $\mathrm{P}$ b .001 . 

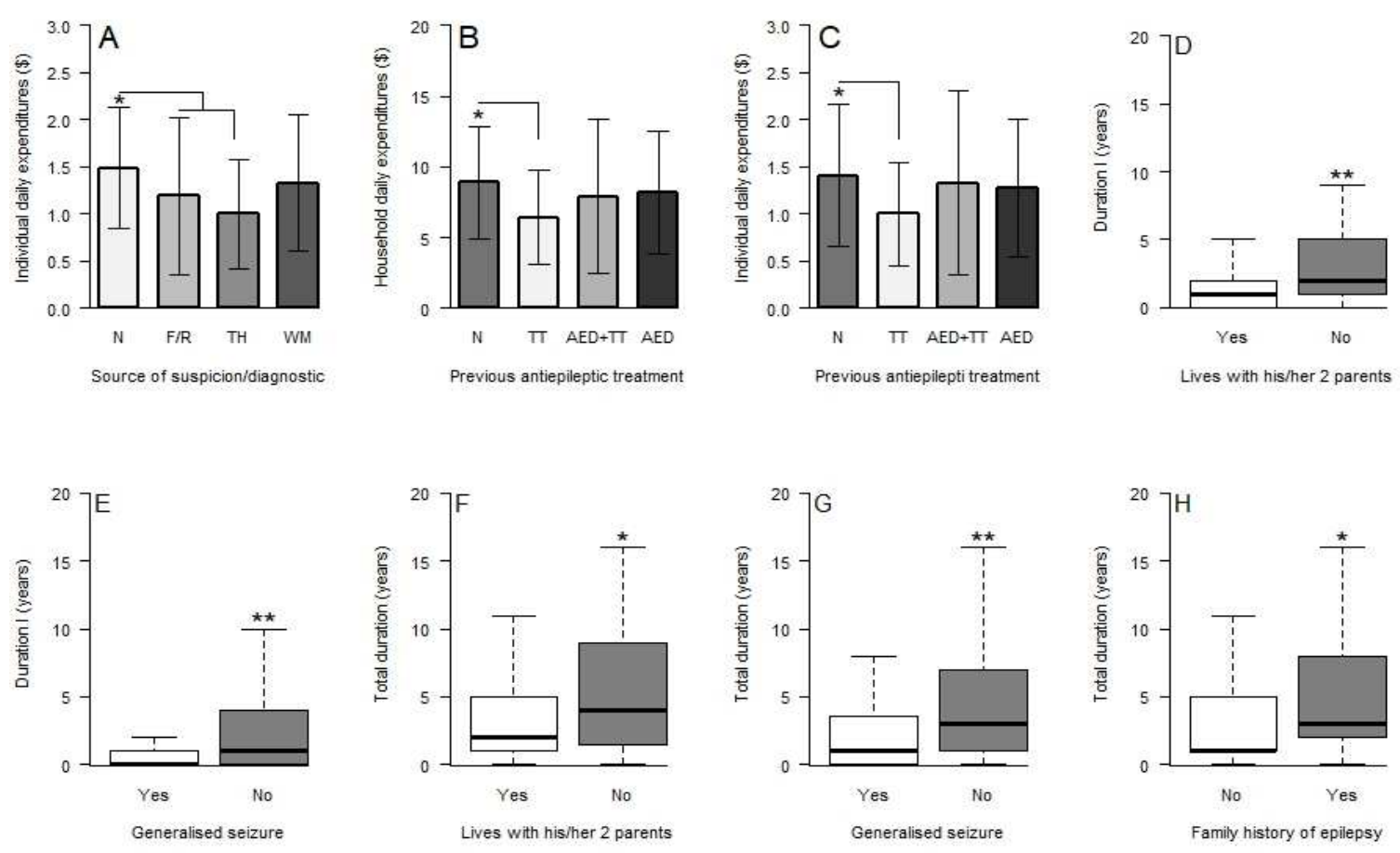

Fig. 2. The features of the therapeutic itinerary and sociodemographic and clinical characteristics of children living with epilepsy. $\mathrm{N}=$ none; $\mathrm{F} / \mathrm{R}=$ family or relatives; $\mathrm{T}=$ traditional healer; $\mathrm{WM}=$ Western medicine. $\mathrm{TT}=$ traditional treatment $\mathrm{AED}+\mathrm{TT}=$ antiepileptic drug combined with traditional treatment; $\mathrm{AED}=$ antiepileptic drug. *: $\mathrm{P}$ b .05; **: P b .01; ***: P b .001. 


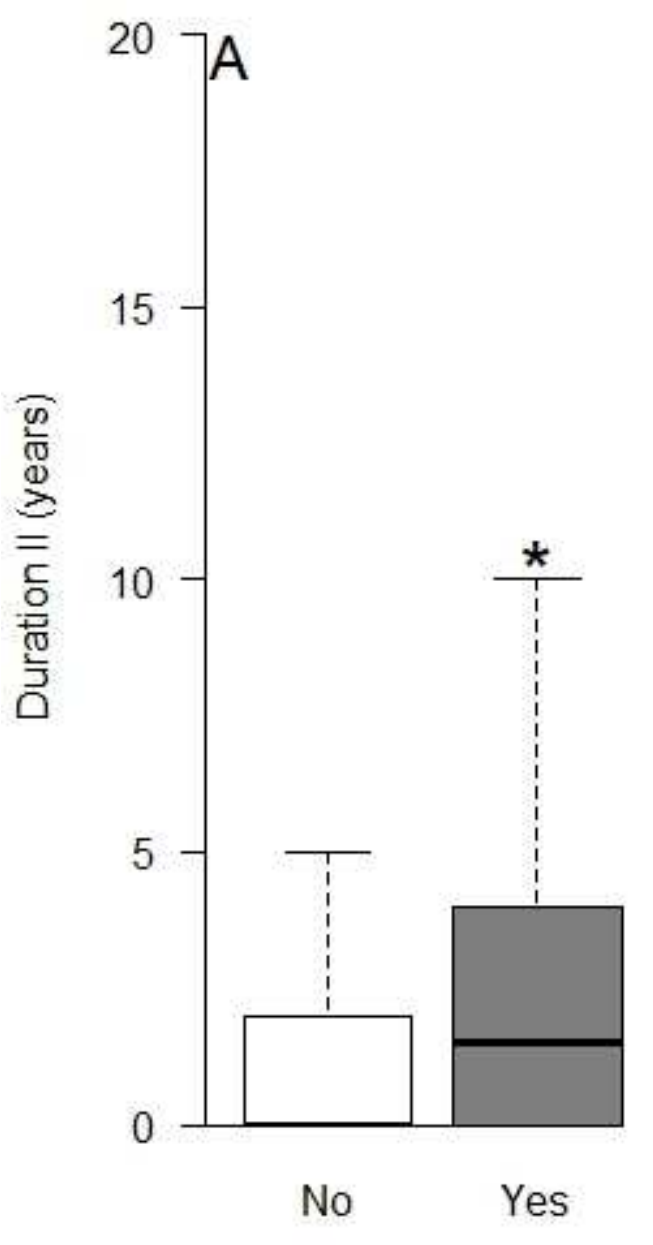

Total behavioral problem

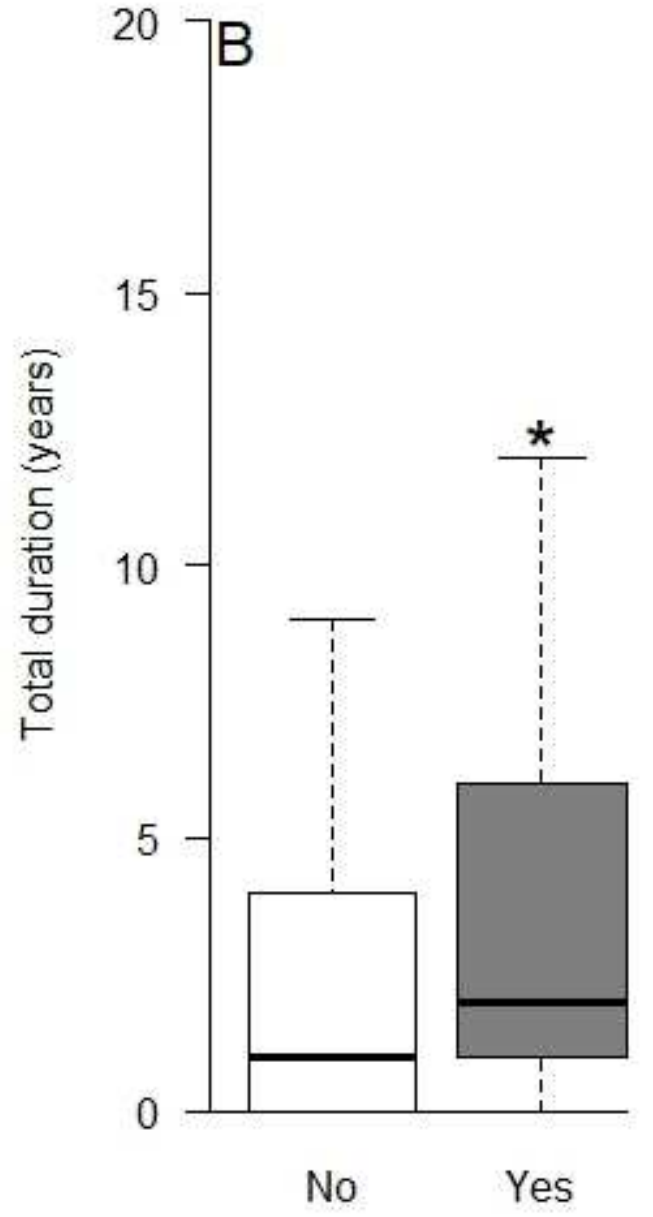

Cognitive impairment

Fig. 3. Behavioral disorder, cognitive impairment, and the duration of therapeutic itinerary. *: $\mathrm{P} \mathrm{b} .05$; **: P b .01; ***: P b .001. 


\section{Supplementary materials}

Table 1. Orthogonal linear contrast codes

\begin{tabular}{lccc}
\hline Variables & Contrast 1 & Contrast 2 & Contrast 3 \\
\hline Source of information & 3 & 0 & 0 \\
- Western medicine & -1 & 2 & 0 \\
- None & -1 & -1 & 1 \\
- Traditional healer & -1 & -1 & -1 \\
- Family/Relatives & & & \\
Antiepileptic treatment & 3 & 0 & 0 \\
- AED & -1 & 2 & 0 \\
- AED - Traditional & -1 & -1 & -1 \\
- Traditional Treatment & -1 & -1 & 1 \\
- None & & & \\
\hline
\end{tabular}

AED: antiepileptic drug. 
Table 2. Source of suspicion or diagnostic and total behavioral problems (with their adjusted residuals)

\begin{tabular}{lccc}
\hline & \multicolumn{2}{c}{ Total behavioral problems } & $\mathrm{P}$ \\
Source of suspicion/diagnostic & Yes & No & .036 \\
\hline None & $8(-0.9)$ & $34(0.9)$ & \\
Family or relative & $\mathbf{1 3 ( 2 . 7 )}$ & $\mathbf{1 9 ( - 2 . 7 )}$ & \\
Traditional healer & $1(-0.8)$ & $7(0.8)$ & \\
Western medicine & $1(-1.7)$ & $14(1.7)$ & \\
\hline
\end{tabular}

